\title{
METAS DE PRODUÇÃO PARA TRABALHADORES DE CORTE FLORESTAL ${ }^{1}$
}

\author{
Amaury Paulo de Souza², Ricardo Brandão Cabral Dutra ${ }^{3}$, Luciano José Minette ${ }^{4}$, Felipe Leitão da Cunha \\ Marzano $^{5}$ e Stanley Schettino 5
}

\begin{abstract}
RESUMO - A quantidade de produção por pessoa por turno de trabalho pode ser questionada pelo empregado, pelo empregador e pelos sindicatos, instituições governamentais e certificadoras. No setor florestal, o processo de determinar metas de produção, em geral, não leva em conta os fatores ergonômicos. O objetivo deste trabalho foi determinar metas de produção para atividade de corte florestal com motosserra (abate e traçamento de árvores), levando em consideração os fatores ergonômicos relacionados à atividade, como forma de prevenir danos à saúde dos trabalhadores. Os fatores ergonômicos foram avaliados utilizando a metodologia de análise ergonômica validada no meio científico. A organização do trabalho foi avaliada segundo a metodologia de análise de sistemas e estudos de tempos. Os resultados indicaram que o nível de vibração total (eixos X, Y e Z), o ambiente térmico expresso pelo Índice de Bulbo Úmido-Termômetro de Globo (IBUTG), o nível de ruído e a carga cardiovascular foram superiores aos limites recomendados para jornada de $8 \mathrm{~h}$ de trabalho. Os movimentos realizados pelos trabalhadores não foram enquadrados como repetitivos. As forças de compressão no disco $\mathrm{L}_{5}-\mathrm{S}_{1}$ da coluna vertebral foram próximas ou superiores ao limite. Com exceção do fator repetitividade, todos os fatores exigiram pausas de recuperação, sendo o nível de vibração da máquina o fator com maior exigência de tempo de pausas. A necessidade de aumentar o tempo de pausa ergonômica e diminuir o tempo de trabalho efetivo fez que a meta de produção fosse reduzida em $18 \%$, para evitar danos à saúde do trabalhador.
\end{abstract}

Palavras-chave: Colheita Florestal; Ergonomia; Produtividade.

\section{PRODUCTION TARGETS FOR WORKERS IN FOREST HARVESTING}

\begin{abstract}
The employee, the employer and labor unions, government institutions and certifiers can question the amount of output per person per workday. In the forestry sector the process of determining production targets, in general, does not take into account the ergonomic factors. The objective of this study was to determine production targets for timber cutting activity with chainsaw (tree felling and bucking) taking into account ergonomic factors related to the activity, as a way to prevent damage to health of workers. The ergonomic factors were assessed using a validated methodology for ergonomic analysis in scientific communities. The work organization was evaluated according to the methodology of systems analysis and time studies. The results indicated that the total vibration level ( $X, Y$, and Z-axis), the thermal environment expressed by the WBGT index (Wet Bulb GlobeThermometer), the noise level, the cardiovascular load exceeded the recommended limits for an eight hour work shift. The movements performed by workers were not classified as repetitive. The forces resulted in compression at $L_{5}-S_{1}$ disc of the spine, near or above the limit. With the exception of repeatability factor, all other factors required fatigue recovery breaks, and the machine vibration level the factor thatrequires more breaks. There was need to increase the ergonomic resting time and consequently to decrease the effective work time, which made the production target reduce $18 \%$, to avoid damage to the workers' health.
\end{abstract}

Keywords: Forest harvesting; Ergonomics; Productivity.

\footnotetext{
${ }^{1}$ Recebido em 17.07.2014 aceito para publicação em 07.05.2015.

${ }^{2}$ Universidade Federal de Viçosa, Centro de Ciências Agrárias, Departamento de Engenharia Florestal, Departamento de Engenharia Florestal, Vicosa, MG - Brasil. E-mail: <amaury@ufv.br>.

${ }^{3}$ Engenheiro de Produção, Quality Assurance Specialist da EMBRACO, Joinville, SC - Brasil. E-mail: <ricardobcdutra@gmail.com>. ${ }^{4}$ Universidade Federal de Viçosa, Centro de Ciências Exatas e Tecnológicas, Departamento de Engenharia Elétrica e de Produção. Campus UFV, Viçosa, MG - Brasil. E-mail: <minette@ufv.br>.

${ }^{5}$ Universidade Federal de Viçosa, Programa de Pós-graduação em Ciência Florestal, Viçosa, MG - Brasil. E-mail: <felipe.marzano@ufv.br> e <stanley.schettino@oi.com.br>
} 


\section{INTRODUÇÃO}

O Brasil é um país com grande cobertura vegetal, e em 2011 as florestas plantadas ocupavam 6,5 milhões de hectares no território brasileiro (ABRAF, 2012). As grandes empresas do setor florestal utilizam alto grau de mecanização, mas ainda há presença, nas empresas menores e mesmo em algumas de grande porte, do processo semimecanizado com a utilização da motosserra para a realização do corte florestal. A remuneração é dada principalmente pela produtividade do trabalhador, gerando grande pressão para que ele produza mais. Segundo Couto (2006), os incentivos de produção, pagos como acréscimo do salário, fazem que muitas vezes o trabalhador exerça sua atividade sentindo dores, somente procurando o serviço médico quando não está mais as suportando, mas nesses casos já pode estar evidente uma doença ocupacional grave.

Há fatores ergonômicos que podem ser modificados para minimizar o impacto que a tarefa exige. Entre esses fatores estão a exposição excessiva ao calor, a vibração e ruído e a realização de posturas inadequadas para executar o trabalho.

No corte florestal, a vibração no trabalho semimecanizado é atrelada ao uso da motosserra, instrumento que tem como característica apresentar altos níveis de oscilações e dificuldade de manuseio. Ela pode causar danos para o sistema vascular, neurológico e musculoesquelético dos membros superiores (NYANTUMBU et al., 2007). O ruído é um fator do trabalho e do cotidiano produzido por máquinas e equipamentos, normalmente associado a som indesejado (ATTWOOD et al., 2004). Quando elevado, é prejudicial à saúde de qualquer pessoa. Para a diminuição dos impactos do ruído no organismo há a necessidade da realização de pausas fora do ambiente de trabalho em ambientes tranquilos.

Trabalhos que envolvem várias posturas e carregamento de peso devem ser avaliados pelo estudo biomecânico. Segundo Vosniak et al. (2010), trabalhadores envolvidos em atividades florestais realizam sua função em ambientes climáticos muito desfavoráveis, utilizando equipamentos que exigem muito esforço e posturas inadequadas.

Os Distúrbios Osteomusculares Relacionados ao Trabalho (DORT) têm causado danos em grande número de trabalhadores que são vítimas de doenças ocupacionais e, em algumas vezes, afastados do seu trabalho por invalidez permanente.

Nesse contexto, o trabalho contínuo e maçante leva à necessidade de pausas, pois esse tipo de trabalho leva acúmulo de ácido lático e dificuldade de circulação do sangue nos tecidos. Com a pausa, haverá o fluxo normal do sangue que irá retirar o ácido lático do músculo, prevenindo as lesões ocupacionais (COUTO, 2006). O gerenciamento do trabalho e a determinação de pausas para os trabalhadores, além de gerar a diminuição nos acidentes e doenças, podem representar um ganho de produtividade do trabalhador e de qualidade no serviço executado, pois este estará trabalhando de acordo com as possibilidades do seu organismo.

As metas de produção ou produtividade do trabalhador sempre foram determinadas em função de estudos de tempos, das variáveis operacionais e de medidas da produção (SANDERS; McCORMICK, 1993; STANTON et al., 2004; VISSER;_SPINELLI, 2012). No entanto, de acordo com Souza et al. (2012), a determinação de metas de produção humanamente sustentáveis deve levar em consideração as limitações físicas e mentais dos trabalhadores. Uma das contribuições da análise ergonômica é a inclusão das questões relativas ao trabalhador, associadas ao estudo de tempos e movimentos.

Nas últimas décadas, o processo de atendimento aos critérios de certificação tem solicitado às organizações a adoção de metas de produção baseadas no amparo científico e legal de condutas que visem à saúde, segurança e bem-estar do ser humano. A determinação de metas de produção baseadas em fatores ergonômicos pode alcançar o que se chama de trabalho humanamente sustentável, considerando que a atividade não compromete a saúde do trabalhador. Essas metas de produção, para serem eficazes e dentro das limitações do trabalho, devem ser feitas através do regime de pausas. O objetivo do trabalho foi determinar metas de produção para atividade de corte florestal com motosserra (abate e traçamento de árvores), levando em consideração os fatores ergonômicos relacionados à atividade, $\mathrm{O}$ objetivo deste trabalho é determinar metas de produção para trabalhadores do corte florestal com motosserra, levando em consideração fatores ergonômicos para proporcionar manutenção da saúde, bem-estar, satisfação e eficiência operacional. 


\section{MATERIAL E MÉTODOS}

\subsection{Local de Estudo}

Para composição do estudo, foram coletados dados e realizadas as análises ergonômicas das atividades de trabalhadores do corte florestal de empresas terceirizadas que prestam serviço para uma empresa de celulose e papel situada na região Centro-Leste do Estado de Minas, situada a 19²2'56" de latitude Sul e 42\%46’33" de longitude Oeste. A região é de clima tropical. Aárea tem altitude de aproximadamente $990 \mathrm{~m}$, a microtopografia da região é marcada por moderadas irregularidades na superfície, com declividade maior que $27^{\circ}$. As coletas de dados foram realizadas no verão, em dias ensolarados e com a presença de ventos moderados.

As atividades dos trabalhadores eram realizadas em povoamentos de eucalipto com 6,85 anos de idade, em média, no período de corte, em espaçamento de $3 \mathrm{~m}$ x 3,33 m. O volume individual médio era de $0,25 \mathrm{~m}^{3}$ por árvore, totalizando $216,87 \mathrm{~m}^{3}$ por hectare. Os trabalhadores envolvidos no estudo tinham idade média de 38 anos, peso médio de $74 \mathrm{~kg}$ e altura média de 1,71 m. A maior parte dos trabalhadores possuía apenas Ensino Fundamental incompleto e já tinha mais de cinco anos de experiência na atividade. O turno de trabalho tinha início às $7 \mathrm{~h}$ da manhã e término às $16 \mathrm{~h}$, com $1 \mathrm{~h}$ de intervalo para almoço e pausas de $15 \mathrm{~min}$ por hora. Os trabalhadores recebiam pagamento adicional por produtividade.

O corte florestal consiste na primeira fase da colheita de madeira, realizado logo após a roçada pré-corte. Era dividido entre as seguintes atividades: derrubada e traçamento, desgalhamento e empilhamento para extração com o guincho. Neste estudo foram contempladas as atividades de derrubada e de traçamento (ambas realizadas pelo mesmo trabalhador). A derrubada e traçamento das árvores eram feitos de forma semimecanizada com o uso de motosserras da marca Stihl, modelo MS 360, pesando $7 \mathrm{~kg}$. Durante essa atividade, o trabalhador caminhava pelo talhão abatendo todas as árvores de uma mesma linha de plantio e retornava pelo talhão abatendo as árvores da linha seguinte. O traçamento consistiu no seccionamento das toras em tamanhos predeterminados por um gabarito de 2,6 m acoplado à motosserra. Essa atividade era realizada pelo próprio operador depois de abater algumas linhas de árvores. Mesmo com a roçada pré-corte sendo executada antes da derrubada, ainda havia a presença de obstáculos para deslocamento como restos de vegetação e pedras.

\subsection{Fatores Ergonômicos}

\subsubsection{Vibração}

As medições de vibração foram feitas com um acelerômetro da marca $01 \mathrm{~dB}-$ Stell Maestro, de acordo com procedimentos técnicos estabelecidos pela Norma de Higiene Ocupacional 10 (FUNDACENTRO, 2013). Essa norma estabelece o limite de exposição ocupacional diária à vibração em mãos e braços (VMB) correspondente a um valor de aceleração resultante de exposição normalizada (aren) de $5 \mathrm{~m} \mathrm{~s}^{-2}$. O instrumento utilizado para medir a aceleração foi da marca $01 \mathrm{~dB}$, modelo Maestro. Para o nível de ação, o aren é igual a 2,5 ms ${ }^{-2}$.

$\mathrm{Na}$ determinação das pausas foram considerados os limites estabelecidos pela American Conference of Industrial Higienists (ACGIH, 2001), que são: aren $<4 \mathrm{~ms}^{-2}$ trabalho contínuo; e $4>$ aren $<64 \mathrm{~ms}^{-2}$ regime de trabalho com $50 \%$ de pausas.

As medições de vibração foram feitas com um acelerômetro da marca $01 \mathrm{~dB}-$ Stell Maestro. Foram medidos os níveis de vibração de mão e braço emitidos pela motosserra, obtendo a aceleração resultante das duas mãos.

\subsubsection{Ruído}

A NR15 regulamentou o nível de exposição do trabalhador ao ruído (BRASIL, 2008). Para uma jornada de $8 \mathrm{~h}$ de trabalho, sendo o máximo estabelecido de $85 \mathrm{~dB}$. O tempo máximo de exposição permitido diminui progressivamente com o aumento do nível de ruído.

Couto (2006), no desenvolvimento do índice de TOR-TOM, estipulou diminuições da porcentagem de trabalho efetivo, conforme a exposição ao ruído, para nível de ruído: abaixo de $80 \mathrm{~dB}(\mathrm{~A})$, não há pausas determinadas; entre $80 \mathrm{~dB}(\mathrm{~A})$ e $85 \mathrm{~dB}(\mathrm{~A}), 2 \%$ de pausas determinadas; entre $86 \mathrm{~dB}(\mathrm{~A})$ e $95 \mathrm{~dB}(\mathrm{~A}), 5 \%$ de pausas determinadas; entre $96 \mathrm{~dB}(\mathrm{~A})$ e $100 \mathrm{~dB}(\mathrm{~A}), 10 \%$ de pausas determinadas; e acima de $100 \mathrm{~dB}$ (A), 15\% de pausas determinadas.

A medição de ruído foi feita com o dosímetro da marca 01dB-Metravib, modelo Wed007, operando no circuito de compensação "A" e circuito de resposta lenta. As leituras foram realizadas com o instrumento 
fixado próximo ao ouvido do trabalhador, durante todo o turno de trabalho.

\subsubsection{Carga de Trabalho Físico}

A frequência cardíaca indica indiretamente a carga física do trabalhador obtida por meio de medidores eletrônicos. A análise da carga cardiovascular com a medição da frequência cardíaca é utilizada para determinar o tempo de pausa durante o turno de trabalho, necessário para a recuperação da fadiga. A metodologia desenvolvida por Bonjer (1974) foi utilizada na determinação da Carga Cardiovascular percentual (CCV). As equações 1, 2 e 3 fazem parte da metodologia.

$$
\mathrm{CCV}=\frac{(\mathrm{FCT}-\mathrm{FCR}) \times(100)}{(\mathrm{FCM}-\mathrm{FCR})}
$$

em que $\mathrm{FCT}=$ frequência cardíaca de trabalho $(\mathrm{bpm})$; $\mathrm{FCR}=$ frequência cardíaca do repouso (bpm); e FCM $=$ frequência cardíaca máxima $(220$-idade $)(\mathrm{bpm})$.

A Frequência Cardíaca Limite (FCL) é obtida pela equação 2 :

$$
\mathrm{FCL}=0,4 \mathrm{X}(\mathrm{FCM}=\mathrm{FCR})+\mathrm{FCR}
$$

Quando a CCV é maior que 40\%, o Tempo de Repouso ( $\mathrm{Tr}$ ) necessário para a realização saudável da atividade é obtido por meio da equação 3 .

$$
\operatorname{Tr}=\frac{\mathrm{Ht} x(\mathrm{FCT}-\mathrm{FCL})}{(\mathrm{FCM}-\mathrm{FCR})}
$$

em que $\mathrm{Ht}=$ duração do trabalho, em minutos.

A frequência cardíaca dos trabalhadores foi medida, durante todo o seu turno de trabalho, utilizando o Monitor de Frequência Cardíaca marca Garmin, modelo Forerunner 305.

\subsubsection{Ambiente Térmico - Calor}

O ambiente térmico foi avaliado com base na norma regulamentadora NR15(BRASIL, 2008). Anorma estabelece que a exposição ao calor deve ser avaliada por meio do Índice de Bulbo Úmido Termômetro de Globo (IBUTG), definido por equações apropriadas. Em função do índice obtido, o regime de trabalho intermitente, com descanso no próprio local de trabalho, é definido para os tipos de atividades leves, moderadas e pesadas. Também, devem ser considerados limites para o regime de trabalho intermitente, com descanso em outro local (local de descanso). O IBUTG foi medido utilizando o medidor da marca Instrutherm, modelo TGD-400.

\subsubsection{Repetitividade}

Segundo Couto (2006), a repetitividade pode ser avaliada considerando os seguintes fatos: número alto de peças concluídas; não existência de pausas curtíssimas; não existência da diversidade dos atos operacionais; existência de movimento que seja bastante repetitivo e tempo de ciclo curtíssimo. Existem mecanismos para recuperação da sobrecarga de trabalho devido à repetitividade, como: atividades de baixa exigência ergonômica, alternância dos grupamentos musculares, pausas, ginásticas laborais e descanso.

$\mathrm{Na}$ análise do trabalho do trabalhador florestal, para o fator repetitividade foi usada a metodologia desenvolvida por Couto (2006), comparando com o estudo de tempos e movimentos desenvolvido. Vídeos obtidos das operações de corte florestal foram utilizados para análise da repetitividade.

\subsubsection{Biomecânica}

A análise biomecânica foi realizada utilizando o software Three-Dimensional Static Strength Prediction Program 3D (SSPP), da Universidade de Michigan, Estados Unidos (THE UNIVERSITY OF MICHIGAN CENTER OF ERGONOMICS, 2005). Os vídeos obtidos durante a realização das atividades permitiram definir as posturas críticas e os ângulos dos segmentos corporais exigidos pelo software. Também, foram medidos os valores das variáveis massa corporal, antropométricas e gênero, além da força exercida pelo trabalhador.

\subsubsection{Estudo de Tempos e Movimentos}

O tempo efetivo de trabalho corresponde ao tempo que o trabalhador está de fato produzindo, descontando todos os tempos improdutivos como as atividades de baixa exigência ergonômica e pausas recomendadas. Atividades de baixa exigência ergonômica são aquelas nas quais o trabalhador não está nem produzindo nem em pausa, como a manutenção do equipamento. O tempo de trabalho efetivo foi obtido pela equação 4 .

$$
T E=T T-T P-T B
$$

em que $\mathrm{TE}=$ Tempo de trabalho efetivo; $\mathrm{TT}=$ Tempo de trabalho total, equivalente à jornada de trabalho; $\mathrm{TP}=$ Tempo de pausas recomendadas; e $\mathrm{TB}=$ Tempo com atividade de baixa exigência ergonômica. 
Para a coleta de dados, o trabalho foi analisado e os tempos de trabalho efetivo, os de pausa e os de atividade de baixa exigência ergonômica foram determinados. As variáveis número de árvores e volume médio por árvore derrubada e traçada por trabalhador foram utilizadas para determinação da produtividade média.

A produção média realizada em volume $\left(\mathrm{m}^{3}\right)$ foi determinada pelo produto entre o número de árvores trabalhadas e o volume médio das árvores.

A nova meta foi determinada de acordo com a proporção do novo tempo efetivo de trabalho recomendado, levando-se em consideração as exigências de pausas relativas aos fatores ergonômicos e o tempo efetivo de trabalho encontrado, como mostrado na equação 5 .

$$
\text { Meta }=\operatorname{Prod} x \frac{\mathrm{TPE}}{\mathrm{TE}}
$$

em que TPE $=$ Tempo efetivo de trabalho considerando as pausas relativas aos fatores ergonômicos; e Prod $=$ Produção média realizada.

\section{RESULTADOS}

Para determinação da meta de produção, os fatores citados anteriormente foram analisados separadamente e, em seguida, identificou-se o que exigia maior tempo de pausa. Esse tempo foi considerado na determinação do novo tempo de trabalho efetivo recomendado.

\subsection{Vibração}

A medição realizada indicou que a aceleração resultante de exposição normalizada (aren) foi igual a $5,80 \mathrm{~m} \mathrm{~s}^{-2}$ para a mão direita e $5,61 \mathrm{~m} \mathrm{~s}^{-2}$ para a mão esquerda. Comparando com os valores encontrados com os limites estabelecidos pela ACGIH, determinase a necessidade de pausas para recuperação dos trabalhadores equivalentes a $50 \%$ da jornada de trabalho.

\subsection{Ruído}

Nas experimentações, o nível equivalente (Leq) de ruído encontrado foi de $94 \mathrm{~dB}$ (A). Como proposto na metodologia do TOR-TOM de Couto (2006), a porcentagem do trabalho destinado às pausas para recuperação devido à exposição ao ruído deve corresponder a $5 \%$ da jornada de trabalho. Além disso, o uso do protetor auricular é obrigatório para a realização da função.

\subsection{Carga de Trabalho Físico}

O tempo de repouso necessário foi determinado considerando o maior valor de carga cardiovascular encontrado entre todos os trabalhadores avaliados. Esse trabalhador tinha 33 anos, frequência cardíaca no repouso igual a $75 \mathrm{bpm}$ e frequência cardíaca de trabalho igual a $134 \mathrm{bpm}$. A carga cardiovascular encontrada foi de $53 \%$, maior que o limite de $40 \%$.

Dessa forma, o tempo de repouso necessário devido a esse fator foi igual a $114 \mathrm{~min}$, correspondendo a $23,75 \%$ da jornada de trabalho.

\subsection{Ambiente térmico - Calor}

A maior média de IBUTG durante a jornada de trabalho, para o intervalo de $60 \mathrm{~min}$, foi de $31^{\circ} \mathrm{C}$. Com os valores considerados para determinação das pausas para a carga de trabalho físico, foi determinada a frequência cardíaca ponderada, que foi igual a $119 \mathrm{bpm}$, que correspondeu a uma atividade mediamente pesada (moderada), cujo IBUTG máximo foi igual a $30^{\circ} \mathrm{C}$. O IBUTG ponderado determinado considerando o local de descanso com IBUTG igual a $22^{\circ} \mathrm{C}$ foi igual a $28,75^{\circ} \mathrm{C}$.

Como o IBUTG ponderado não ultrapassou o máximo, o regime de pausas, em local de temperatura mais amena, de 15 min para cada hora trabalhada, era adequado para a atividade.

\subsection{Repetitividade}

A análise das filmagens realizadas durante a jornada de trabalho permitiu a obtenção das seguintes informações: o número de peças prontas por turno não ultrapassa os 8.000 , as pausas curtíssimas não ultrapassam os $15 \%$ do ciclo, os atos operacionais são diversificados, nenhum desses é repetido mais do que 3.000 vezes por turno e o tempo do ciclo é maior do que $30 \mathrm{seg}$. Portanto, seguindo essa metodologia, não há necessidade de pausas adicionais devidas ao fator repetitividade.

\subsection{Biomecânica}

Para a análise feita pelo 3D-SSPP da Universidade de Michigan, o limite máximo de força de compressão do disco vertebral $\mathrm{L}_{5}-\mathrm{S}_{1}$ é de $3.426 \mathrm{~N}$. A Tabela 1 apresenta a análise feita a partir do programa para quatro posturas diferentes. Apenas uma posição ultrapassa o limite permitido. A segunda posição analisada, mesmo não gerando alto valor para a força de compressão no disco 
$\mathrm{L}_{5}-\mathrm{S}_{1}$, gera alto risco para o joelho do trabalhador. Dessa forma, o treinamento postural dos trabalhadores deve orientar para que sejam adotadas as posturas que representam menor risco de lesão para o trabalhador. Nesse caso, a primeira posição é a indicada para a derrubada, e a quarta posição é a indicada para o traçamento de árvores.

\subsection{Estudo de Tempos e Movimentos}

A análise dos tempos e movimentos no trabalho do operador de motosserra forneceu os seguintes valores médios: Tempo de Trabalho Efetivo igual a $292 \mathrm{~min}$
(60,83\% da jornada), Tempo de Pausas Recomendadas igual a 59 min (12,29\% da jornada) e Atividade de Baixa Exigência Ergonômica igual a 129 min (26,88\% da jornada). Com esse regime de pausas, cada trabalhador era capaz de derrubar e traçar, em média, 132 árvores por dia. Como o volume considerado foi de $0,25 \mathrm{~m}^{3}$ por árvore, a produtividade do trabalhador era, em média, de $33 \mathrm{~m}^{3}$ por jornada de trabalho.

\subsection{Determinação da meta de produção}

Analisaram-se todos os fatores ergonômicos e, para determinação do tempo de trabalho efetivo recomendado, foi levado em consideração o que exigiu

Tabela 1 - Risco de Lesão das Articulações e Força de Compressão no disco vertebral L5-S1 para cada postura, de acordo com o software 3D-SSPP de Michigan.

Table 1 - Risk of Joints Injury and Compression Force on spinal disc $L_{5}-S_{1}$ for each posture, according to the Michigan 3D SSPP software.

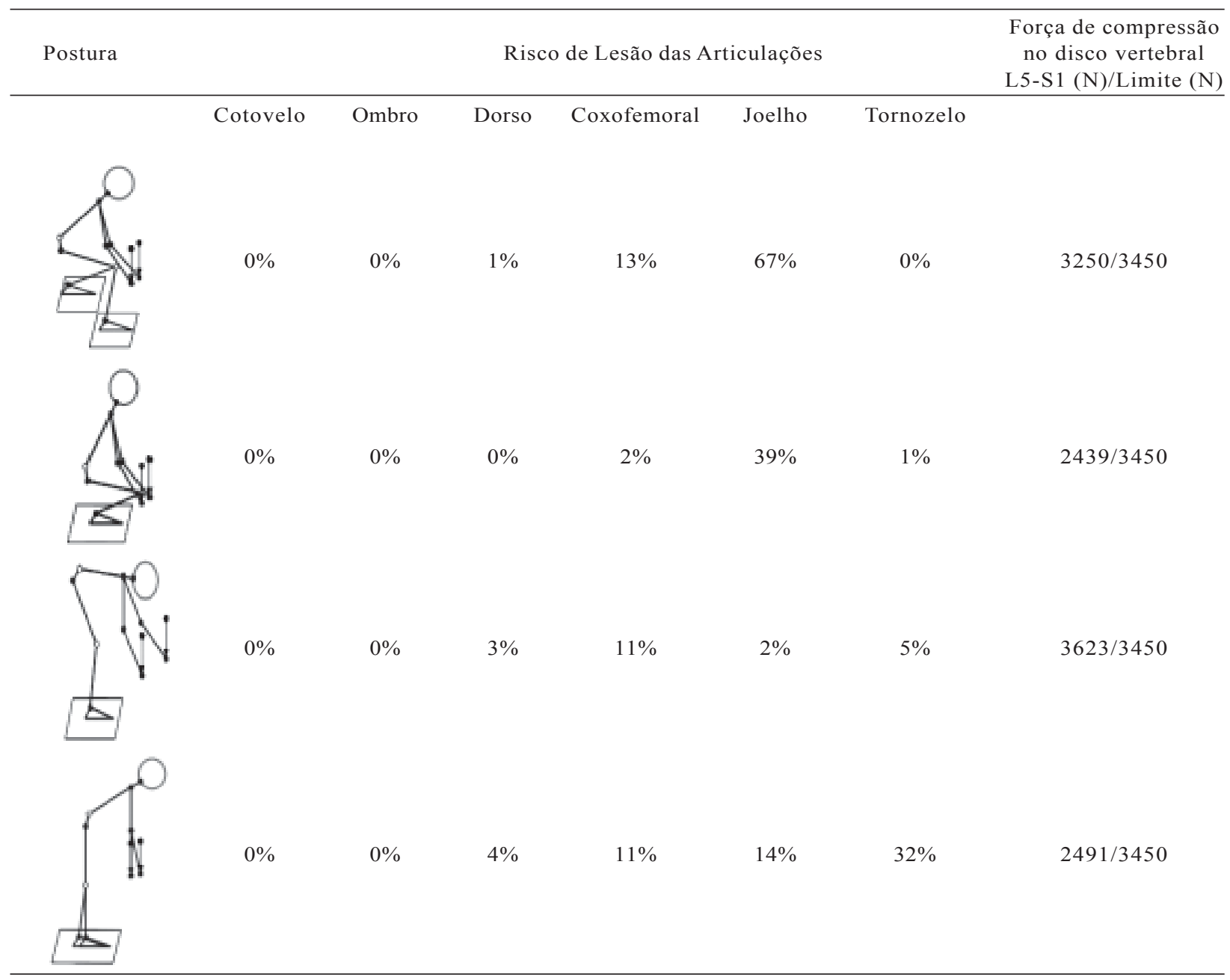

Revista Árvore, Viçosa-MG, v.39, n.4, p.713-722, 2015 
maior tempo de repouso. Os fatores ergonômicos foram analisados na Tabela 2, de acordo com a conformidade da exposição deles durante toda a jornada de trabalho e a porcentagem de pausas que esse fator exigiu para a recuperação do trabalhador.

O tempo de pausas e de atividades de baixa exigência ergonômica correspondeu a 39,17\% da jornada de trabalho. Esse tempo é inferior ao tempo de pausas exigido pelo fator principal, que é a vibração, equivalente a $50 \%$ da jornada de trabalho. Na situação analisada, a produtividade diária média era de $33 \mathrm{~m}^{3}$ de madeira por trabalhador, considerando um tempo de trabalho efetivo equivalente a $60,83 \%$ da jornada. Como o tempo de trabalho efetivo recomendado é igual a 50\% da jornada, a nova meta de produção diária não deve ultrapassar os $27,12 \mathrm{~m}^{3}$ de madeira por trabalhador.

\section{DISCUSSÃO}

Os resultados indicaram que a meta de produção para trabalhadores de corte florestal estabelecida, em conformidade com os princípios ergonômicos, deve ser o objeto dos responsáveis pelo planejamento da operação para obter o equilíbrio da performance humana em sistema pessoa-máquina.

Na colheita de madeira, geralmente as pesquisas focam a produção ou a produtividade do trabalhador em função dos fatores florestais, operacionais e edáficos. Vários autores (VISSER; SPINELLI, 2012; MAESANO et al., 2013; NUTTO et al., 2013) abordaram diferentes fatores que influenciam a produtividade sem levar em consideração os fatores humanos. Outros estudaram a influência de fatores isolados na performance humana (LUNDGREN et al., 2014).
Na área de silvicultura e colheita de madeira, pesquisas foram realizadas englobando os principais fatores ergonômicos: ambiente térmico, pressão sonora, vibração, carga de trabalho físico, repetitividade e organização do trabalho (SOUZA et al., 2012; SOUZA et al., 2015). Os fatores limitantes da produtividade foram: ambiente térmico, vibração e carga de trabalho físico.

Gallis (2013), em sua pesquisa “aumento de produtividade e controle de fatiga do trabalho em operações florestais por meio de pausas", concluiu que pausas ativas de 10 min podem aliviar operadores florestais dos efeitos da fadiga, permitir tempo para a recuperação e manter a adaptação ao trabalho.

Johnson et al. (2001), avaliando a performance humana em trabalho de manutenção de aviação, relataram que os fatores duração do sono, temperatura ambiente, pressão sonora e iluminação tiveram efeito na performance e não estavam de acordo com os limites.

O fator que exigiu maior tempo para a recuperação do trabalhador foi a vibração. De acordo com os resultados, o tempo máximo de operação da motosserra não deveria ultrapassar $50 \%$ da jornada de trabalho, para evitar riscos de surgimento da síndrome da vibração mão-braço. Nesse caso, a produção do operador por turno de trabalho foi reduzida, porém os riscos de dano à saúde foram minimizados. Além do efeito sobre a saúde, essa síndrome causa grande prejuízo para a qualidade de vida dos portadores (HOUSE et al., 2014). O uso de motosserras com dispositivos antivibratórios de alta performance contribui para minimizar o efeito desse fator na saúde do trabalhador e, assim, obter ganhos de produtividade.

Tabela 2 - Conformidade e porcentagem de pausas determinadas para cada fator ergonômico analisado. Table 2 - Conformity and percentage of breaks determined for each ergonomic factor analyzed.

\begin{tabular}{lcc}
\hline Fator ergonômico & $\begin{array}{c}\text { Conformidade } \\
\text { (Sim, não, mudar postura) }\end{array}$ & $0,00 \%$ \\
\hline Repetitividade & Sim & $23,75 \%$ \\
Carga de Trabalho Físico & Não & Não \\
Vibração (NR 15) & Não & Não \\
Vibração (ACGIH) & Não & $25,00 \%$ \\
Calor & $5,00 \%$ \\
Ruído & Mudar postura \\
Biomecânica (3D SSPP) & - \\
Fator crítico: vibração (ACGIH) & $50,00 \%$ \\
\hline
\end{tabular}


O segundo fator mais importante na determinação da meta da meta de produção foi o esforço físico com carga cardiovascular superior ao limite recomendado. Também, Çalýskan e Çaglar (2010), em pesquisas realizadas em operações de corte florestal com motosserra, na Turquia, encontraram valores superiores aos limites, sendo as atividades classificadas com trabalho pesado e a produtividade, reduzida.

O ambiente térmico avaliado por meio do IBUTG foi o terceiro fator, em ordem de importância, na determinação de metas de produção com base na necessidade de pausas de regulação fisiológica. $\mathrm{O}$ trabalho a céu aberto em dias ensolarados, nos meses de verão, as temperaturas diárias no local de trabalho (principal variável na determinação do índice) eram elevadas e o consequente IBUTG (Alfredo, este trecho está confuso). A legislação brasileira sobre segurança do trabalho fornece limites e regime de pausas de descanso conforme a carga de trabalho físico e valores de IBUTG. O regime de pausas na época do estudo atendia a normas. Lundgren et al. (2014), analisando estresse causado pelo calor associado com a redução de produtividade, utilizando o modeloPredicted Heat Strain (PHS), concluíram que valores altos de IBUTG tiveram significante impacto na produtividade, em todos os locais de trabalho.

As posturas críticas adotadas pelos trabalhadores na realização da atividade representavam risco de lesões corporais, portanto a meta de produção determinada somente podia ser atingida após a alteração das posturas que afetavam as articulações coxofemorais, joelho, tornozelo e o disco intervertebral (L5-S1).

A meta de produção para trabalhadores da operação de corte florestal avaliada foi reduzida em, aproximadamente, 18\%, com base nos princípios ergonômicos. A prescrição de metas de produção com amparo científico gera possibilidade de a empresa calcular a sua capacidade de produção real e, assim, posicionar-se no mercado, pois, sabendo os limites físicos da sua mão de obra, a empresa poderá determinar quanto ela pode produzir com segurança e preparar-se para atender à sua demanda. Além disso, as empresas poderão ter a confiança de que estão agindo de acordo com o que a legislação trabalhista exige, podendo, assim, buscar certificações que melhoram a imagem e o prestígio da empresa com a sociedade e os seus clientes.

Revista Árvore, Viçosa-MG, v.39, n.4, p.713-722, 2015

\section{CONCLUSÕES}

A análise da operação de corte florestal, com enfoque na atividade de derrubada e traçamento, permitiu concluir que a produção diária do trabalhador excedeu a recomendada, considerando as exigências dos principais fatores ergonômicos do trabalho.

A repetitividade foi o único fator ergonômico analisado que estava em conformidade com os limites de exposição durante toda a jornada de trabalho. Os fatores ruído, vibração, carga de trabalho físico e calor não estavam em conformidade, exigindo pausas de recuperação do trabalhador.

As posturas críticas adotadas pelos trabalhadores na realização da atividade representavam risco de lesão corporal, portanto a meta de produção determinada somente podia ser atingida após a alteração das posturas que afetavam as articulações coxofemorais, o joelho, o tornozelo e o disco intervertebral (L5-S1).

O fator ergonômico vibração foi o que exigiu maior percentual de pausa em relação ao tempo da jornada, portanto foi o fator determinante para o estabelecimento da nova meta de produção compatível com as limitações físicas do trabalhador.

\section{AGRADECIMENTOS}

Ao Conselho Nacional de Desenvolvimento Científico e Tecnológico (CNPq), pelas Bolsas de Produtividade em Pesquisa, Bolsas de Iniciação Científica e fornecimento de recursos para realização de pesquisas; à Fundação de Amparo à Pesquisa do Estado de Minas Gerais (FAPEMIG), pelo apoio financeiro para compra de equipamentos; ao Laboratório de Ergonomia (LABERGO) da Universidade Federal de Viçosa (UFV), pelo apoio com estrutura e aparelhagem necessárias.

\section{REFERÊNCIAS}

ABRAF. Anuário Estatístico: ano base 2011/ABRAF. Brasília: 2012. 130p.

AMERICAN CONFERENCE OF INDUSTRIAL HIGIENISTS. Hand-arm vibration. In: ACGIH. Documentation of the threshold limit values for physical agents. 7.ed. Cincinnati: 2001.

ATTWOOD, D.A.; DEEB, J.M.; DANZ-REECE, M.E. Ergonomic solutions for the process industries. Oxford: Elsevier, 2004. 459p. 
BONJER, F.H. Energy expenditure. In: INTERNATIONAL Labour office. Enciclopedia de medicina, hygiene y seguridade del trabajo. Madrid: INP, 1974. v.1. p.750-760.

BRASIL. Ministério do Trabalho e Emprego. NR 15 - Atividades e Operações Insalubres. In: SEGURANÇA e medicina do trabalho. 2.ed. São Paulo: Saraiva, 2008. 1041p.

COUTO, H.A. Índice TOR-TOM: Indicador ergonômico da eficácia de pausas e outros mecanismos de regulação. Belo Horizonte: Ergo, 2006. 335p.

ÇALISKAN, E.; ÇAGLAR, S. An assessment of physiological workload of forest workers in felling operations. African Journal of

Biotechnology, v.9, n.35, p.5651-5658, 2010.

FUNDACENTRO (BRASIL). Norma de Higiene Ocupacional 10: Procedimento Técnico. Avaliação da Exposição Ocupacional a Vibrações em Mãos e Braços. São Paulo: 2013. 53p.

GALLIS, G. Increasing productivity and controlling of work fatigue in forest operations by using prescribed active pauses: a selective review. Croatian Journal of Forest Engineering, v.34, n.1, p.103-112, 2013.

HOUSE, R.; WILLS, M.; LISS, G.; SWITZERMCINTYRE, S.; JIANG, D. The effect of hand-arm vibration syndrome on quality of life.

Occupational Medicine, v.64, n.2, p.133135,2014

JOHNSON, W.B.; HALL, S.; WATSON, J. Evaluation of aviation maintenance: working environments, fatigue, and human performance. Egg Harbor Township: Galaxy Scientific Corporation, 2001.37p.

LUNDGREN, K.; KUKLANE, K.; VENUGOPAL, V. Occupational heat stress and associated productivity loss estimation using the PHS model (ISO 7933): a case study from workplaces in Chennai, India. Global Health Action, v.7, p.25362, 2014.

MAESANO, M.; PICCHIO, R.; MONACO, A.L.; LASSERRE, F.N.B.; MARCHETTI, M. Productivity and energy consumption in logging operation in a Cameroonian tropical forest. Ecological Engineering, v.57, August, p.149-153, 2013.

NYANTUMBU, B.; BARBER, C. M. ROSS, M.; CURRAN, A. D; FISHWICK, D.; DIAS, B.; KGALAMONO, S.; PHILLIPS, J. I. Hand-arm vibration syndrome in South African gold miners. Occupationl Medicine, v.57, n.1, p.25-29, 2007.

SANDERS, M.M.; MCCORMICK, E.J. Human factors in engineering and design. 7.ed. New York: Mcgraw-Hill, 1993.

SOUZA, A.P.; MINETTE, L.J.; SANCHES. A.L.; SILVA, E.P.; RODRIGUES, V.A.; OLIVEIRA, L.A. Ergonomic factors and production target evaluation in eucalyptus timber harvesting operations in mountainous terrains. Work, v.41, Suppl.1, p.4957-4962, 2012.

SOUZA, A.P.; MARZANO, F.L.C.; SCHETTINO, S.; MINETTE, L.J. Production targets consistent with ergonomic factors of forestry activities. In: AREZES, P. M.; BAPTISTA, J. S.; BARROSO, M. P.; CARNEIRO, P. CORDEIRO, P.; COSTA, N.; MELO, R. B.; A. MIGUEL, A. S.; PERESTRELO, G. (Ed.) Occupational safety and hygiene III. Boca Raton: CRC Press, 2015. p. 71-74.

STANTON, N.A.; HEDGE, A.; KAREL BROOKHUIS, K.; SALAS, E.; HENDRICK, H. W. (Ed.) Handbook of human factors and ergonomics methods. Boca Raton: CRC Press, 2004.

\section{THE UNIVERSITY OF MICHIGAN CENTER OF} ERGONOMICS. 3D Static Strength Prediction Program Version 6.0.5.: Manual's user. Michigan: The Regents of The University of Michigan, 2011. 108p.

VISSER, R.; SPINELLI, R. Determining the shape of the productivity function for mechanized felling and felling-processing. Journal of Forest Research, v.17, n.5, p.397-412, 2012.

VOSNIAK, J.; LOPES,E.S.; FIEDLER, N.C.; ALVES, R.T.; VENÂNCIO, D.L. Carga de trabalho físico e postura na atividade de coveamento semimecanizado em plantios florestais. Scientia Forestalis, v.38, n.88, p.589-598, 2010.

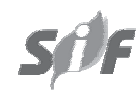

Revista Árvore, Viçosa-MG, v.39, n.4, p.713-722, 2015 
NUTTO, L.; MALINOVSKI R. A.;

BRUNSMEIER M.; SANT'ANNA F. S.

Ergonomic aspects and productivity of different pruning tools for a first pruning lift of Eucalyptus grandis Hill ex Maiden. Silva Fennica, v. 47, n. 4, p. 1-10, 2013. 